\title{
RELACIONES ENTRE LOS CAMPOS Y LAS CATEGORÍAS EDUCACIÓN Y TECNOLOGÍA: UN ANÁLISIS DESDE UNA PERSPECTIVA HISTÓRICO-CULTURAL*
}

\author{
José Gabriel Cristancho Altuzarra ${ }^{1}$
}

\begin{abstract}
RESUMEN: El objetivo de este texto fue analizar las relaciones entre los campos y las categorías educación y tecnología. Metodológicamente, el trabajo se orientó por una perspectiva histórico-cultural, reconociendo que los conceptos y campos se han construido socialmente en contextos histórico-políticos. El artículo se configura en tres partes; en primer lugar, se ocupa de una primera relación entre educación y tecnología que se configuró entre los siglos XVII y XIX. En la segunda parte, se analiza cómo en el siglo XX la relación entre educación y tecnología adquirió unas especificidades marcadas por las dos guerras mundiales, la Guerra Fría y el inicio de la globalización; en la tercera parte, se muestran algunas tensiones que esto ha implicado en el siglo XXI; se cierra con perspectivas para futuros trabajos.
\end{abstract}

Palabras clave: Educación. Tecnología. Historia de la educación. Ciencia. Sociedad del conocimiento.

\section{RELATIONS BETWEEN EDUCATION AND TECHNOLOGY FIELDS AND CATEGORIES: ANALYSIS FROM A HISTORICAL AND CULTURAL OUTLOOK}

ABSTRACT: The purpose of this article was to analyze the relationships between education and technology fields and categories. Methodologically, the research assumed a historical and cultural perspective, recognizing that the concepts and categories were socially developed based on historical and political contexts. This paper is divided into three parts: the first one analyzes the first relationship between education and technology configured during the $17^{\text {th }}$ and $19^{\text {th }}$ centuries. The second part shows how this relationship acquired distinctive characteristics related to the two world wars, the Cold War, and the beginning of the Globalization in the $20^{\text {th }}$ century. The third part shows some of the tensions that it implied in the $21^{\text {st }}$ century. The text ends with perspectives for future investigations.

Keywords: Education. Technology. History of education. Science. Knowledge society.

\footnotetext{
*El artículo se realizó en el marco del proyecto de investigación Usos pedagógicos de las Tecnologías de la Información y la Comunicación de la Universidad Cooperativa de Colombia seccional Bogotá, financiado por el Consejo Nacional de Investigaciones (CONADI) de esa institución. ${ }^{1}$ Universidad Cooperativa de Colombia; Universidad Pedagógica Nacional - Bogotá, Colombia.

E-mail: jogacral@outlook.com

DOI: 10.1590/ES0101-73302016148243
} 


\section{RELATIONS ENTRE LES DOMAINES ET LES CATÉGORIES ÉDUCATION ET TECHNOLOGIE: ANALYSE DANS UNE PERSPECTIVE HISTORIQUE ET CULTURELLE}

RESUME: L'objectif de cet article est d'analyser quelles étaient les relations entre les domaines et les catégories éducation et technologie. Méthodologiquement cette recherche a été guidée par une perspective historique-culturel, en reconnaissant que les concepts et les domaines ont été socialement construits dans des contextes historiques et politiques. Cette étude a été configuré en trois parties; tout d'abord, on s'occupe d'une première relation entre l'éducation et la technologie configuré entre les XVIIe et XIXe siècles. La seconde partie explique comment la relation entre l'éducation et la technologie dans le XXe siècle a acquis des particularités marquées par de deux guerres mondiales, la guerre froide et le début de la mondialisation; dans la troisième et dernière partie, le texte montre certaines tensions du XXIe siècle, et on ferme le texte avec des perspectives de travail.

Mots-clés: Éducation. Technologie. Histoire de l'éducation. Science. Société de la connaissance.

\section{Introducción}

$\mathrm{L}$

os avances tecnológicos actuales son tan vertiginosos que cada vez más parece insoslayable que la educación esté actualizada. Así, en las últimas décadas se ha acrecentado el interés por establecer políticas y agendas académicas sobre la alfabetización tecnológica para incrementar los conocimientos y las habilidades en el uso de las Tecnologías de la Información y la Comunicación -TIC (AGUADED-GÓMEZ, 2012; GUTIÉRREZ; TYNER, 2012), por la búsqueda de mejoras de los usos de las TIC como herramientas pedagógicas (PRIETO, 2001; MCCLOSKEY, 2012; ZAPATA-ROS, 2013), la educación virtual como modalidad de educación a distancia (MACKNESS; MAK; WILLIAMS, 2010; BRESLOW et al., 2013), entre otras. Por estas circunstancias, se han dado estudios encaminados a satisfacer estas demandas (OILO, 1998; GROSSO, 2004; SCHALK, 2010) y se han construido los estándares de competencias en las TIC para docentes (UNESCO, 2011).

Esos intereses y las investigaciones relacionadas son fundamentales para garantizar un mayor y mejor acceso a las potencialidades que ofrecen la tecnología en general y las TIC en particular. No obstante, parecen dejar en segundo plano los estudios que propendan por la actitud vigilante y crítica frente a las causas y secuelas de esos procesos. Otros autores han asumido esa senda propiciando debates en torno a las implicaciones sociales y culturales sobre una posible mirada acrítica de estas tendencias (MARTÍN-BARBERO, 2009), pero mostrando las 
potencialidades que brinda a los proyectos educativos contraculturales (RUEDA; QUINTANA, 2013, p. 28; 90).

En este sentido, este artículo busca renovar la cuestión, máxime porque las relaciones entre estos campos y conceptos no han sido suficientemente problematizadas a pesar de los trabajos referenciados ${ }^{1}$. Por ello, el objetivo de este texto es presentar resultados parciales de una indagación que busca ampliar el debate examinando cuáles han sido las relaciones que han existido entre la educación y la tecnología como campos y categorías para comprenderlas en la época contemporánea ${ }^{2}$. Metodológicamente, la indagación se orientó con una perspectiva histórico-cultural reconociendo que todo concepto y campo es fruto de prácticas y expresiones culturales y, por lo tanto, ha sido construido socialmente, anclado en procesos y contextos históricos y políticos.

El artículo se estructura en tres partes: en la primera se plantea que, entre los siglos XVIII y XIX y en el marco del proyecto político y cultural de la modernidad, se encuentran antecedentes fundamentales de lo que hoy se concibe como educación y tecnología; en la segunda parte, se explicita el desarrollo de esa relación inicial tomando en cuenta el contexto del siglo XX. En la tercera, se muestran las tensiones que han implicado el avance de la tecnología y la aparente permanencia de la educación a referentes tradicionales.

\section{La ciencia moderna y la matriz de una relación embrionaria: escuela y técnica}

Para entender la relación entre la educación y la tecnología que se ha ido tejiendo en la época contemporánea, es necesario considerar las circunstancias históricas y culturales que fueron dando forma a los sistemas educativos y a la ciencia moderna entre los siglos XVII y XIX. A lo largo de estos siglos otras categorías, como institutio, instrucción e ilustración, fueron el germen de la categoría y el campo de la educación, el cual hace parte del proyecto trazado por la modernidad y que se materializa en la escuela como institución (NOGUERA, 2012; CRISTANCHO, 2013).

En el proceso de construcción de los Estados (ANDERSON, 2006), se fue configurando la educación como campo, es decir, como espacio-temporalidad, desde donde se ha ejercido y hacia donde confluyen ciertas acciones sociales, para formar las identidades nacionales (HOBSBAWM, 1998; CARRETERO; CASTORINA, 2010). En esa medida, la escuela pública se estableció para la formación moral y política que contribuyera al desarrollo económico de los Estados por medio del conocimiento y uso de ciencias útiles (MARTÍNEZ-BOOM, 2012; CRISTANCHO, 2013).

En este contexto, se va perfilando lo que podría considerarse la relación embrionaria entre la educación y la tecnología, que se tejería entre la escuela y la técnica, mediada por la ciencia moderna. En efecto, las modificaciones sustan- 
ciales que desde el siglo XVII o antes venían teniendo la concepción y el uso del conocimiento, dieron pie al surgimiento de ciencias concentradas en objetos específicos y al interés por hacer del conocimiento algo útil y práctico que beneficiara social y económicamente a las sociedades (SHAPIN, 1994).

Por eso, el acceso a la instrucción deja de ser un privilegio de las élites para ser un derecho y una obligación (MARTÍNEZ-BOOM, 2012; CRISTANCHO, 2013), lo que implicó la reestructuración de las universidades para incluir las nuevas ciencias y la paulatina creación de otras escuelas superiores y politécnicas que fueron integrando las escuelas de artes y oficios. Si bien la categoría tecnología no existía, en virtud de los desarrollos de las ciencias y la educación en investigación científica y sus aplicaciones fueron propiciándose los avances técnicos que darían pie a la revolución industrial en el siglo XIX y que serían la base de lo que luego se llamaría tecnología ${ }^{3}$.

Uno de los intelectuales que en esa época plantea estas conexiones es Comte; para él, "el progreso constituye, tanto como el orden, las condiciones fundamentales de la civilización moderna" (COMTE, 1958, p. 103). Insiste en que la educación ha de ayudar a consolidar en todos los seres humanos el "espíritu positivo" (COMTE, 1958, p. 128-129), pues si esto se da a todas las inteligencias

sentirán la misma necesidad fundamental de esa filosofía primera, que resulta del conjunto de las necesidades reales y que debe, pues llegar a ser la base sistemática de la razón humana, tanto activa como especulativa, para cumplir más convenientemente el indispensable cometido que tuvo en otro tiempo la universal instrucción cristiana. (COMTE, 1958, p. 142).

Comte realizó una propuesta de formación escolar que implica que la educación sea ejercida especialmente a los proletarios, para que en ellos se elimine todo rezago de superstición. Con esto se instituye la enseñanza de un relato histórico, el del espíritu positivo; gracias a esto, dice Comte, "los fenómenos resultan así cada vez más modificables, ofreciendo un dominio cada vez más basto a la intervención humana" (COMTE, 1958, p. 174).

El mismo investigador complementa esta reflexión de carácter teórico que sitúa a la escuela en ese papel, con otro elemento de importancia peculiar: la vida industrial posibilita el espíritu positivo en la medida en que tiene una eficacia especulativa inherente a ella sobre todo en los operarios (COMTE, 1958 , p. 148), porque, para Comte, ellos viven de salario, son la mayoría de la población, sus funciones se reducen a ejecutar y no se preocupan por el fin de sus acciones. De ahí que sea esto situado como una condición del espíritu positivo. En este modo de vida del operario - tan inmediato, tan lleno de necesidades - , se configura una mirada en la que todo debe estar en orden a lo inmediato — no a lo abstracto —, a lo práctico — no a lo especulativo —, y a 
lo básico - no a las causas finales. En una forma de vida así, los operarios no verán, ni sentirán la necesidad de reflexionar sobre sus acciones, ni de buscar un sentido profundo o más allá de lo inmanente a ellas: "Se trata, en efecto, de asegurar convenientemente a todos, en primer término, la educación normal, luego el trabajo regular: tal es en efecto, el programa social de los proletarios" (COMTE, 1958, p. 156).

Así, Comte (1958) realizó unas reflexiones sobre el orden de cosas de su tiempo, pero las articuló con un proyecto político y cultural que implica una concepción del mundo natural y social en sintonía con los procesos industriales y una concepción de lo educativo pensado para articularse con esa concepción. Si bien Comte (1958) no plantea que la educación sea para producir la técnica sí la plantea como una educación para producir "el espíritu positivo", una categoría que podríamos asumir como manera de situarse ante el mundo (CRISTANCHO, 2013, p. 68) es decir, un ethos que propicia el uso técnico de los mundos natural y social. En esta propuesta, Comte ve como procesos formativos de ese ethos tanto la educación formal en la ciencia (escuela), como el trabajo cotidiano de la industria, para aprender un modo de saber operativo y práctico radicalmente opuesto al saber que él denomina especulativo.

Se haya tenido en cuenta o no lo proyectado por Comte, se fue configurando, no sin tensiones sociales, un modo de entender y modelar la educación concentrada en la formación de sujetos que pudieran estar dedicados al cultivo de nuevas ciencias experimentales modernas o, al menos, que pudieran usar el conocimiento en función del aumento de la riqueza de las naciones. Ese interés aunque fuera común a los contextos nacionales, tenía sus matices y diferencias específicas, pero esta relación se mantendría prácticamente a lo largo de los siglos XIX y XX, configurando de manera complementaria los sistemas educativos y el desarrollo científico y tecnológico en cada Estado según sus avances y procesos sociales e históricos que implicaron estos dos siglos.

\section{El desarrollo de la relación educación y tecnología en el siglo XX}

La relación embrionaria adquirió unas especificidades a lo largo del siglo XX que sólo pueden caracterizarse de manera pertinente tomando en cuenta las dos guerras mundiales, la Guerra Fría y el contexto contemporáneo de la globalización. La experiencia de las dos guerras mundiales y la Guerra Fría se desarrollaron a escalas inimaginables, gracias a los avances técnicos y tecnológicos. El sistema burocrático en torno a los asesinatos en serie, los aparatos técnicos y tecnológicos diseñados para ese fin y los experimentos indiscriminados sobre personas pusieron de presente el poder y el horror de los usos de la tecnología (BIRDSALL, 2012). Lo perpetrado por el nazismo antes y durante la Segunda Guerra Mundial llevó a que Adorno dijera que: 
La exigencia de que Auschwitz no se repita es la primera de todas en la educación. Hasta tal punto precede a cualquier otra que no creo deber ni poder fundamentada. No acierto a entender que se le haya dedicado tan poca atención hasta hoy (ADORNO, 1998, p. 79).

Esta exigencia se extiende no obstante no sólo respecto de lo sucedido en Auschwitz, sino a todos los usos propiciados o auspiciados por la tecnología, como los seńalados por Weindling (1994), Alexander (2006) y Fridlund (2011). El uso de la energía nuclear implicó la detonación de la bomba atómica por parte de Estados Unidos sobre Hiroshima y Nagasaki, con efectos devastadores sobre la población civil, y fue el abrebocas de la continua carrera armamentista entre los Estados Unidos y la Unión Soviética en su disputa por la hegemonía mundial en la Guerra Fría.

Estas circunstancias generaron fuertes cuestionamientos como los planteados por Marcuse para quien:

El método científico que lleva a la dominación cada vez más efectiva de la naturaleza llega a proveer así los conceptos puros tanto como los instrumentos para la dominación cada vez más efectiva del hombre por el hombre a través de la dominación de la naturaleza. [...] Hoy, la dominación se perpetúa y se difunde no sólo por medio de la tecnología sino como tecnología, y la última provee la gran legitimación del poder político en expansión, que absorbe todas las esferas de la cultura. (MARCUSE, 1993, p. 184-185).

Marcuse señaló que la ciencia moderna posee un carácter interno instrumentalista "gracias al cual es una tecnología a priori, y el a priori de una tecnología específica; esto es, una como forma de control social y de dominación" (MARCUSE, 1993, p. 185); así, con el tono propio de la teoría crítica, Marcuse pone de presente la lógica de la dominación que implica la racionalidad tecnológica, extensión o consumación de la racionalidad científica.

Aunque Marcuse no cita directamente el tema de la educación, da cuenta de uno de los modos como estaba interpretándose en su tiempo el predominio de la ciencia y de lo tecnológico sobre lo social y su incidencia en la configuración de los sujetos, asunto deseado por Comte en el siglo XIX. Ambos planteamientos tienen su respectivo tono ideológico; lo relevante es que se prospecta (Comte) o se pondera (Marcuse) el lugar decisivo de la técnica y la tecnología en las sociedades, emparentado con los procesos sociales e históricos que implicaban la emergencia y paulatina consolidación del capitalismo y en el que la escuela y la ciencia tienen un lugar peculiar.

En efecto, por un lado, el auge y los avances de las ciencias experimental y aplicada y el desarrollo de la tecnología llevaron a pensar que la educación también podría ser planeada y ejecutada desde sus principios; así, el auge de la psicología 
conductista y el enfoque técnico-racional de la primera mitad del siglo XX fueron dándole forma a la denominada tecnología educativa (AREA, 2009, p. 16-17).

En el siglo XX, las relaciones entre la educación y la tecnología fueron configurándose inicialmente en dos formas complementarias: en la primera se asume la tecnología como el conjunto de instrumentos o dispositivos con los cuales se busca implementar o mejorar el proceso educativo en los escenarios escolares, o lo que podría denominarse la 'pedagogización de instrumentos tecnológicos'; en la segunda, la tecnología es asumida como un modo eficaz de proceder, de tal suerte que se buscan modos y pasos necesarios para hacer del ejercicio educativo un proceso eficiente en el alcance de unos objetivos previamente diseñados y planeados (RUEDA; QUINTANA, 2013, p. 75), o lo que podría denominarse 'tecnologización de la educación'.

A lo largo del siglo XX, la pretensión de tecnologizar la educación propició cierta polarización en diversas manifestaciones culturales: por un lado, algunos intelectuales estimularon esta perspectiva, principalmente desde la psicología conductista (SKINNER, 1968; 1973); otros plantearon sus críticas como Anthony Burguess desde la literatura (BURGESS, 1986; 2012) y la versión cinematográfica de su novela, realizada por Stanley Kubrick (KUBRICK, 1971).

No obstante, la aparición del ordenador y de otros instrumentos tecnológicos parecieron dar un rumbo prometedor a la tecnología educativa, sobre todo del lado de la pedagogización de los instrumentos tecnológicos y de una nueva figura que conformaría una tercera forma complementaria de relación entre la educación y la tecnología: la tecnología asumida como un componente disciplinar que debe ser integrado en el currículo como área específica, pero también como un componente transversal en el currículo presente en cada área; lo que podríamos denominar la "curricularización de la tecnología".

Para finales la década de los 1980 y a lo largo de los 1990, esto se afianzó más con los cambios geopolíticos que se han ido consolidando en el siglo XXI, los cuales se condensan en lo que se ha denominado globalización. Como fenómeno sociológico, la globalización se ha desarrollado simbióticamente con la tecnología. No obstante, las urgencias cada vez más vertiginosas de la globalización parecen ocluir sus rasgos históricos, así como las tensiones que han generado entre la educación y la tecnología en el siglo XXI.

\section{Tensiones entre la educación y la tecnología en el siglo XXI}

Las urgencias cada vez más vertiginosas de la globalización parecen ocluir sus rasgos históricos, los cuales no se limitan a los avances de las ciencias y la tecnología, sino que incluyen el paso del orden bipolar de la Guerra Fría a un mundo unipolar (MOUFFE, 2007, p. 14), la consolidación del neoliberalismo 
como modelo administrativo-político que se dio desde fines de los ańos 1970 del siglo pasado y la caída de la Unión Soviética a finales del siglo XX.

Por lo tanto, es necesario aclarar que la globalización es un denso fenómeno político y sociocultural de complejas redes reales y virtuales que propician por y se sostienen en el continuo intercambio de capitales económicos, culturales y sociales y en el que las fronteras tradicionales de los Estados-nación son reconfiguradas de muros a canales de interacción para la circulación e incremento de todo tipo de capital; un proceso y producto por el cual el capitalismo postindustrial se ha ido configurando como proyecto económico y político de orden mundial ${ }^{4}$.

En estas circunstancias, que han marcado profundamente el siglo XXI, la globalización se ha desarrollado simbióticamente con la tecnología gracias a sus desarrollos en los ámbitos en los que destacan las TIC; en este marco, el interés de relacionar la educación y la tecnología se ha dado no desde meras iniciativas de los Estados-nación, como fuera la relación embrionaria.

En efecto, como bien lo señalan otros autores (TARABINI; BONAL, 2011), el contexto de la globalización exige que la educación sea pensada y estudiada no únicamente desde contextos locales o nacionales, sino en relación con intereses de diversas instituciones que después de la Segunda Guerra Mundial han venido configurando el orden mundial (UNESCO, OCDE, FMI, Banco Mundial), y las necesidades sociales a las cuales los sujetos deben responder para vivir mejor o, en el peor de los casos, sobrevivir.

La permanente actualización y difusión de conocimientos y habilidades que la tecnología posibilita en el marco de la globalización implica mayores y mejores accesos de distintos capitales culturales y materiales para sujetos individuales y colectivos. Por ello, se han configurado los sistemas de innovación, ciencia y tecnología (SNCYT), que aunque son de índole nacional ${ }^{5}$, pretenden fortalecer la investigación y las redes de trabajo colaborativo en el marco de las necesidades globales de producción de conocimiento.

Como efecto de estas políticas, determinados métodos y problemas de investigación se van posicionando por encima y en detrimento de otros, superponiendo campos del saber y modos de conocimiento que requieren ser más estudiados y conocidos. La investigación y el uso de la energía nuclear han avanzado pese a los riesgos que no han podido evitar accidentes como el de la central nuclear de Chernóbil en 1986 y el de Fukushima I en Japón en 2011, asunto que genera grandes controversias. También hay incentivos cada vez mayores a las investigaciones en campos de investigaciones genética y biotecnológica, a pesar de las controversias éticas y culturales sobre la racionalidad científica y tecnológica interviniendo sobre los distintos ámbitos de la vida, o por la propiedad intelectual de los "descubrimientos" científicos que pretenden superponerse frente a sociedades tradicionales (GREENE, 2006; POHLENZ, 2009; DOMÍNGUEZ, 2006; DÍAZ, 2013). 
Además, al mismo tiempo que se propicia el auge de la tecnología, no todos los sujetos tienen los mismos accesos y van quedando excluidos de recursos económicos y culturales. En el tercer mundo y en particular en Latinoamérica, las exclusiones y brechas tecnológicas han sido puestas en evidencia por diversos autores (RUEDA; QUINTANA, 2013; DUSSEL, 2010; CALVO; ROJAS, 2007).

Aun así se sigue viendo como necesidad adquirir ciertos conocimientos o habilidades mínimas en la tecnología para desenvolverse en las sociedades contemporáneas. De este modo, no sólo parece tener sentido que exista una relación entre educación y tecnología sino una justificación contextual. Estas apuestas están incidiendo directa o indirectamente en las políticas de educación superior y, por lo tanto, en las políticas de educación en general ${ }^{6}$.

Lo que se ha expuesto hasta aquí llama la atención sobre cómo los campos de la educación y la tecnología emergen anclados al proyecto de la modernidad, se entrecruzan y complementan. Los avances científicos van propiciando lo que Comte había proyectado y lo que Marcuse había diagnosticado, o sea la técnica y la tecnología pueden ser concebidas como dos dimensiones: la dimensión instrumental o procedimental y la dimensión estructural o modo de ser de las sociedades (MARTÍN-BARBERO, 2006, p. 13). No obstante, mientras la escuela surgió como una institución disciplinaria que apela a ciertos referentes tradicionales, la técnica ha devenido tecnología y sus avances vertiginosos generan tensiones entre estos campos.

Una primera tensión tiene que ver con las formas de la construcción de conocimiento. Para Dussel, las formas de construir el conocimiento en las TIC

funcionan en base a la personalización, la seducción y el involucramiento personal y emocional, y suelen ser muy veloces y con una interacción inmediata. La escuela, en cambio, es una institución basada en el conocimiento disciplinar, más estructurada, menos exploratoria, y con tiempos y espacios determinados de antemano, más lentos y menos porosos. (DUSSEL, 2010, p. 13).

Para Martín-Barbero, en virtud de las mediaciones tecnológicas, el saber se descentra de sus ejes de referencia como son el libro y el maestro, que fueron las autoridades tradicionales del conocimiento (MARTÍN-BARBERO, 2003, p. 81-85).

La segunda tensión se deriva de la anterior y ocurre en las tres relaciones entre la educación y la tecnología que se configuraron desde el siglo XX y que se expusieron en el anterior acápite: la pedagogización de instrumentos tecnológicos, la tecnologización de la educación y la curricularización de la tecnología. Estas no son excluyentes sino complementarias y tienen como común denominador la tensión de que la tecnología como campo parece subsumir a la educación, en tanto que ésta debe asumir la forma, los contenidos y procesos que aquella implica. 
Las anteriores tensiones emergen y convergen en una tercera, relacionada con la importancia que en la educación, como proyecto político y cultural, se le da a los conocimientos teorético y técnico. Para entender la cuestión es necesario tener presente la antigüedad, cuando se reivindicaba el conocimiento contemplativo, en detrimento de la experiencia. Así lo plantearía Platón en la alegoría de la caverna (PLATÓN, 1988, p. 338-343); Aristóteles no sería tan radical pues reconocía el conocimiento empírico, otorgándole un lugar a la techné pero dejándola en posición inferior respecto de los conocimientos teoréticos (Figura 1).

La valoración de los conocimientos teórico o contemplativo por encima de los técnicos implicó la configuración de un proyecto educativo que se extendió a lo largo de la Edad Media, distinguiéndose entre las artes liberales y manuales $\mathrm{u}$ oficios (ABBAGNANO; VISALBERGHI, 1992). Frente a la estructura escolástica, en la que se cultivaron las artes liberales, Descartes planteó sus cuestionamientos (cf. nota 3) y frente a los rezagos de ese proyecto se va lanza en ristre Comte.

En ambos proyectos educativos (el antiguo y el moderno) se supone una oposición entre el conocer y el hacer; cada concepción y proyecto educativo responde a dos tipos de racionalidad que aparentemente se oponen: la humanista y la técnica. Actualmente esta oposición se ha afianzado más en beneficio de la segunda y en detrimento de la primera, en virtud de que en el campo de la educación, desde finales de los 90, se ha configurado un modelo de formación que, con pretensión de universalidad, ha venido institucionalizándose y naturalizándose; se trata del modelo de formación por competencias.

En efecto, desde fines del siglo XX, la Comisión Internacional sobre la Educación para el siglo XXI (UNESCO, 1996) propugna por un modelo de formación centrado en el hacer y en las habilidades operativas, concepto que luego tomaría forma con la categoría competencias (skills o competences en inglés) desarrollado por el organismo multilateral, la Organización para la Cooperación y el Desarrollo (OCDE u OECD, por sus siglas en inglés) (SALGANIK et al. 1999; ANANIADOU; CLARO, 2009; OECD, 2012).

\begin{tabular}{|c|c|c|c|}
\hline & & sophia (SABIDURÍA) & \multirow{2}{*}{$\begin{array}{c}\text { Conocimientos teóricos } \\
\text { theôrêtikai }\end{array}$} \\
\hline & & episteme (CIENCIA) & \\
\hline & techné & jefes de obra; obreros & \multirow{3}{*}{$\begin{array}{c}\text { Conocimientos prácticos } \\
\text { poiêtikón }\end{array}$} \\
\hline & \multicolumn{2}{|c|}{ EXPERTICIA } & \\
\hline \multicolumn{3}{|l|}{ EXPERIENCIA } & \\
\hline
\end{tabular}

Figura 1

Estratificación de los tipos de conocimiento en la Metafísica, 981a (ARISTÓTELES, 2012, p. 2-3). Elaboración propia. 
La formación por competencias reivindica el saber hacer, con lo cual pretende ejercer una síntesis conceptual entre conocimiento teórico y aplicación del mismo (técnica y tecnología). Reivindicando una tercera dimensión de la formación (la actitudinal, que según esta apuesta, corresponde al ser), se pretende apuntar a la formación integral; este horizonte se ha configurado como política educativa global y su hegemonía cultural ha venido imponiéndose paulatinamente en diversas instituciones de educación básica, media y superior de diversas partes del mundo.

Hay una línea de continuidad argumentativa en el informe de la Organización de las Naciones Unidas para la Educación, la Ciencia y la Cultura (UNESCO) y los trabajos de la OCDE, con la que se hace especial énfasis para justificar, tanto el modelo de formación por competencias como las reformas que ello implica; se argumenta la necesidad de establecer más relaciones entre educación y empresa, o como lo dice explícitamente uno de los informes: "ser pertinente es estar en contacto con el mundo del trabajo" (UNESCO, 1998, p. 20).

Esta insistencia se entronca con la necesidad de ser competitivo en el mercado global; si bien la categoría competitividad proviene de la economía, ésta se ha extendido a otros ámbitos de la existencia humana, de tal suerte que también a la universidad y sus miembros se le exige esta característica (UNESCO, 1998; BENEITONE et al. 2007). La competitividad, entendida como la capacidad de ganar una posición destacada en un contexto de disputa o rivalidad para la consecución de un fin, se configura en un horizonte cultural y político. A este interés por que todos los sujetos, comunidades y organizaciones posean esta característica en el mercado global se le denomina en este artículo "racionalidad competitiva", la cual puede ubicarse en un amplio marco económico y político como lo es capitalismo contemporáneo y la globalización.

Con lo que fue expuesto en este artículo, se sostiene la hipótesis de que el horizonte de formación por competencias sería la síntesis y consumación de que la tecnología - entendida en sentido amplio, más que como un conjunto de instrumentos, como una racionalidad específica — , subsuma a la educación, en tanto que ésta asume la forma, los contenidos y procesos que la tecnología implica, subsunción que se manifiesta en que el eje central de la educación del ser humano es lo que podría denominarse la configuración del sujeto tecnologizado (conectado y competente) en el marco de la racionalidad competitiva.

Estos planeamientos abren un profundo interrogante sobre el horizonte de formación humanístico y crítico que apunta a otras dimensiones prácticas, a saber las dimensiones ética y política. No se trata de demonizar el campo de la tecnología sino de problematizar su hegemonía y en este aspecto los aportes de Sennett contribuyen en esta dirección ${ }^{7}$.

$\mathrm{Su}$ trabajo se contrapone a los planteamientos de Arendt, quien indicó una dicotomía entre el animal laborans y el homo faber (ARENDT, 1993). Para la 
filósofa, el animal laborans es incapaz de pensar la pregunta por el por qué y el para qué de su hacer, cuestión que sí se plantea el homo faber. Así, al humano que ejerce techné, al artesano, no le va bien tomar decisiones. Eso le corresponde al homo faber, al político, quien, una vez vistos los efectos negativos de la técnica, ha de direccionar el pasivo rol mecánico del artesano.

Para Sennett, esta división es falsa

porque menosprecia a la persona práctica volcada en su trabajo. El animal humano que es el animal laborans tiene capacidad de pensar; el productor mantiene discusiones mentales con los materiales mucho más que con otras personas (SENNETT, 2009, p. 18).

Además, la dimensión lúdica, creativa y estética de la artesanía impulsan a Sennett a inquirir "qué nos enseńa de nosotros mismos el proceso de producir cosas concretas” (SENNETT, 2009, p. 19).

Sennett declara que: “Artesanía' designa un impulso humano duradero y básico, el deseo de realizar bien una tarea, sin más” (SENNETT, 2009, p. 20). Desde esta perspectiva, su estudio lo lleva a rescatar al animal laborans y a establecer una conexión entre la dimensión práctica técnica, que se evidencia en el ejercicio de la artesanía, con las dimensiones práctica moral y política, evidente en el ejercicio de la ciudadanía, conexión que era imposible en el planteamiento de Arendt y en la tradición humanista. Él parte de la idea de que:

El artesano explora las dimensiones de habilidad, compromiso y juicio de una manera particular. Se centra en la estrecha conexión entre la mano y la cabeza. Todo buen artesano mantiene un diálogo entre unas prácticas concretas y el pensamiento; este diálogo evoluciona hasta convertirse en hábitos, los que establecen a su vez un ritmo entre la solución y el descubrimiento de problemas. (SENNETT, 2009, p. 21).

De este modo, Sennett insinúa que "un buen artesano necesariamente es un buen ciudadano" si tiene iniciativas en la vida pública y participa en las decisiones con miras a la solución de problemas públicos; se percibe una crítica a todas las políticas que han implicado ver al artesano como mero animal laborans, ente mecánico sometido al homo faber en la lógica de la competitividad. Esto sugiere a las sociedades contemporáneas que configuren una mixtura de la educación artesanal y la ciudadana.

De lo anterior se infiere que existe una relación entre el hacer ético y político del ser humano y el estético y técnico; por lo tanto, estos dos tipos de hacer constituyen la dimensión práctica del ser humano ${ }^{8}$; además, la escisión entre conocer y hacer, o si se quiere, entre teoría y práctica, es insulsa. No puede afirmarse que la racionalidad tecnológica sea per se la causa de todos los males, pues es fruto de un 
modo existencial propio del ser humano: la artesanía. Pero también cuestiona que la tecnología subsuma a la educación y que la racionalidad humanista y crítica tenga que ceder a la formación y práctica de un tipo de pensamiento ágil y operativo.

\section{Perspectivas}

El recorrido del artículo ha logrado mostrar que las relaciones entre los campos y las categorías educación y tecnología no han sido suficientemente problematizadas. Orientándose por una perspectiva histórico-cultural, se plantea que entre los siglos XVII y XIX se configuró la relación embrionaria entre educación y tecnología: la articulación escuela-técnica direccionando la ciencia al provecho de las economías nacionales.

Se mostró luego que en el siglo XX la relación entre educación y tecnología adquirió unas especificidades marcadas por las dos guerras mundiales, la Guerra Fría y el contexto contemporáneo de la globalización; éste último fenómeno generó que la tecnología como campo parezca subsumir a la educación, en tanto que ésta se ve obligada a asumir la forma, los contenidos y procesos de aquella. Esto puede ser el síntoma o efecto de formas obtusas de concebir el conocer y el hacer que han provocado la escisión de lo técnico frente a lo ético y lo político, al tiempo que la escisión entre la teoría y la práctica.

Se mostró que, en la época contemporánea, existe un horizonte de formación que pretende la formación integral argumentando además su necesidad en el contexto de la globalización: la formación por competencias; no obstante, se planteó la hipótesis de que el horizonte de formación por competencias podría ser la síntesis y consumación de que la tecnología — entendida en sentido amplio, más que como un conjunto de instrumentos, como una racionalidad específica -, subsuma a la educación, en tanto que en este modelo de formación el eje central de la educación del ser humano es lo que podría denominarse, la configuración del sujeto tecnologizado (conectado y competente) en el marco de la racionalidad competitiva.

Este trabajo reabre el debate en torno a la necesidad de hacer un beneficio de inventario de la tecnologización de la educación, de configurar apuestas teóricas alternativas frente a la conceptualización de teoría y práctica, del conocer y del hacer, que hagan justicia a estas categorías y a la perspectiva crítica frente al contexto de la globalización y de abrir el interrogante acerca de qué otros horizontes de formación alternativos asumen una respuesta a los retos actuales buscando la transformación del orden social.

Existe otro horizonte de formación que en sus nodos fundamentales le interesa la articulación entre el conocer y el hacer, entre la teoría y la práctica y que además cuestiona el orden de cosas que ha implicado la globalización: la pedagogía crítica. De este modo, la pregunta por este horizonte y su posición frente a la formación por competencias y las relaciones entre la educación y la tecnología son de obligado estudio en próximos trabajos. 


\section{Notas}

1. Un importante antecedente es el planteamiento de Rueda y Quintana (2013, p. 41-94), y su recorrido que va desde la filosofía de la tecnología dando cuenta de distintas tradiciones, hasta mostrar algunos rasgos de categorías como educación en tecnología, tecnología en educación y tecnología educativa.

2. Esta indagación hace parte de la construcción de los fundamentos teóricos del proyecto de investigación Usos pedagógicos de las Tecnologías de la Información y la Comunicación de la Universidad Cooperativa de Colombia seccional Bogotá, financiado por el Consejo Nacional de Investigaciones (CONADI) de esa institución en el marco de las pesquisas del grupo de investigación CIDU.

3. Como proyecto esto había sido avizorado desde Descartes: "Tan pronto como adquirí nociones generales relativas a la física [...], vi hasta dónde podían conducirnos [...]. Estas nociones me hicieron ver que es posible llegar a la adquisición de conocimientos utilísimos para la vida, y que en lugar de la filosofía especulativa que se enseña en las escuelas, se puede encontrar una filosofía eminentemente práctica, por la cual, conociendo la fuerza y las acciones del fuego, del agua, del aire, de los astros, de los cielos y de todo lo que nos rodea, tan distintamente como conocemos los oficios de nuestros artesanos, aplicaríamos esos conocimientos a los objetos adecuados y nos constituiríamos en señores y poseedores de la Naturaleza”. (DESCARTES, 2001, p. 38).

4. Sobre el concepto globalización y la densa discusión en torno a la categoría, véase Beck (1998) y Reich (1998).

5. Sobre los SNCYT en América Latina y el Caribe, véase Lemarchand (2010).

6. Un breve ejemplo de la cuestión es lo que plantea Colciencias, SNCYT de Colombia: que el sistema educativo requerido hoy es de "ciudadanos integrales, con capacidad de innovar y con capacidad de adaptarse de forma rápida y eficaz a los cambios, con capacidad de emprendimiento que permite convertir los nuevos conocimientos en innovaciones exitosas" (COLCIENCIAS, 2013, p. 2).

7. Sennett se propone desarrollar su estudio en tres libros, de los cuales este artículo se concentra en el primero (El Artesano): "Éste es el primero de tres libros sobre cultura material [...]. Los tres atañen a la cuestión de la técnica, pero entendida más como asunto cultural que como procedimiento irreflexivo; cada uno se refiere a una técnica para llevar un modo de vida particular". (SENNETT, 2009, p. 20).

8. Diversos pensadores (KANT, 2008; ARENDT, 1993; HABERMAS, 1986) se refieren a lo práctico relacionándolo sólo con lo ético y político y diferenciándolo de lo técnico y estético. Como se expuso mediante el planteamiento de Sennett, dicha escisión en la categoría omite las dimensiones ética y política de la estética y técnica y viceversa.

\section{Referencias}

ABBAGNANO, N.; VISALBERGHI, A. Historia de la pedagogía: Madrid: Fondo de cultura Económica, 1992.

ADORNO, T.W. Educación para la emancipación. Conferencias y conversaciones con Hellmut Becker (1959-1969). Madrid: Morata, 1998. 
AGUADED-GÓMEZ, J. I. Apuesta de la ONU por una educación y alfabetización mediáticas. Comunicar, Huelva, v. 19, n. 38, p. 7-8, mar. 2012. Disponible en: <http:// www.revistacomunicar.com>. Acceso el: 30 en. 2014.

ALEXANDER, J. An efficiency of scarcity: using food to increase the productivity of Soviet prisoners of war in the mines of the Third Reich. History and Technology: An International Journal, v. 22, n. 4, p. 391-406, nov. 2006. Disponible en: <http://www.tandfonline.com/ doi/abs/10.1080/07341510601003107?.journalCode=ghat20\#.VRrTovyUdRk $>$. Acceso el: 12 feb. 2013.

ANANIADOU, K.; CLARO, M. 21st century skills and competences for new millennium learners in OECD countries. EDU Working paper no. 412009. 2009. Disponible en: <http://www.oecd.org/officialdocuments/publicdisplaydocumentpdf/? cote=EDU/ WKP\%282009\%2920\&doclanguage=en $>$. Acceso el: 12 ene. 2014.

ANDERSON, B. Comunidades imaginadas. Reflexiones sobre el origen y la difusión del nacionalismo. México: Fondo de Cultura Económica, 2006.

AREA, M. Introducción a la tecnología educativa. San Cristóbal de la Laguna: Universidad de La Laguna, 2009. Disponible en: < https://campusvirtual.ull.es/ocw/file.php/4/ebookte. pdf>. Acceso el: 20 mar. 2014.

ARENDT, H. La condición humana. Barcelona: Paidós, 1993.

ARISTÓTELES. Metafísica. Madrid: Gredos, 2012.

BECK, U. ¿Qué es la globalización? Barcelona: Paidós, 1998.

BENEITONE, P. et al. Reflexiones y perspectivas de la educación superior en América Latina. Informe final Proyecto Tuning. América Latina 2004-2007. Bilbao: Universidad de Deusto; Universidad de Groningen, 2007.

BIRDSALL, C. Nazi Soundscapes: Sound, Technology and Urban Space in Germany, 1933-1945. Amsterdam: University Press, 2012.

BRESLOW, L.; PRITCHARD, D. E.; DEBOER, J.; STUMP, G. S.; HO, A. D.; SEATON, D. T. Studying learning in the worldwide classroom: Research into edx's first MOOC. Research \& Practice in Assessment, v. 8, p. 13-25, 2013. Disponible en: <http:// www.rpajournal.com/dev/wp-content/uploads/2013/05/SF2.pdf>. Acceso el: 12 ene. 2014.

BURGESS, A. A clockwork orange. Middlesex: Penguin Books, 1986.

. La naranja mecánica. Barcelona: Minotauro, 2012.

CALVO, A.; ROJAS, S. Exclusión social y tecnología. Comunicar, Huelva, v. 15, n. 29, p. 143-148, 2007. Disponible en: <http://www.revistacomunicar.com>. Acceso el: 21 feb. 2014.

CARRETERO, M.; CASTORINA, A. La construcción del conocimiento histórico. Buenos Aires: Paidós, 2010.

COLCIENCIAS. Modelo de medición de grupos de investigación, desarrollo tecnológico e innovación. Bogotá: Departamento Administrativo de Ciencia, Tecnología e Innovación, 2013. Disponible en: $\quad$ http://www.colciencias.gov.co/sites/default/files/ckeditor files/files/DOCUMENTO\%20 Modelo\%20de\%20Medici\%C3\%B3n\%20Grupos\%202013-VERSI\%C3\%93N\%20II\%20 Definitiva\%20DIC\%2010\%202013\%20 protected.pdf $>$. Acceso el: 12 ene. 2014. 
COMTE, A. Discurso sobre el espíritu positivo. Buenos Aires: Aguilar, 1958.

CRISTANCHO, J. Algunos diálogos sobre educación. Medellín: Universidad de Antioquia, 2013.

DESCARTES, R. Discurso del método. México: Porrúa, 2001.

DÍAZ, G.L. Saber tradicional y conocimiento cientifico: debate por la Propiedad Intelectual. Ponencia presentada en IX Congreso Nacional e Internacional de Sociología y Ciencias Sociales. 2013. Disponible en <http://docplayer.es/13159928-Saber-tradicional-yconocimiento-cientifico-debate-por-la-propiedad-intelectual.html>. Acceso el: 7 mar. 2014.

DOMÍNGUEZ, J. Un acercamiento a la patentabilidad de las invenciones biotecnológicas. Revista Facultad de Derecho y Ciencias Políticas, Medellín, v. 36, n. 104, p. 111-136, 2006. Disponible en: <http://www.redalyc.org/articulo.oa?id=151413538005>. Acceso el: 7 mar. 2014.

DUSSEL, I. Aprender y enseñar en la cultura digital. Buenos Aires: Santillana, 2010.

FRIDLUND, M. Buckets, bollards and bombs: towards subject histories of technologies and terrors. History and Technology: An International Journal, v. 27, n. 4, p. 391-416, 2011. Disponible en: <http://www.tandfonline.com/doi/pdf/10.1080/07341512.2011.622149>. Acceso el: 11 ene. 2014.

GREENE, S. ¿Pueblos indígenas S.A.? La cultura como política y propiedad en la bioprospección farmacéutica. Revista Colombiana de Antropologia, Bogotá, v. 42, p. 179221, 2006. Disponible en: <http://biblioteca.icanh.gov.co/DOCS/MARC/texto/REV0915-v42a07.pdf>. Acceso el: 7 mar. 2014.

GROSSO, J. L. NTICs, educación virtual, lógicas de la comunicación social y "sociedad del conocimiento": anotaciones sobre el contexto y la responsabilidad social de la educación superior a distancia en ALC. Diálogo Educacional, Paraná, v. 4, n. 13, p. 1-10, 2004. Disponible en: <http://www.redalyc.org/articulo.oa?id=189117791006 >. Acceso el: 5 mar. 2014.

GUTIÉRREZ, M.; TYNER, K. Alfabetización mediática en contextos múltiples. Comunicar, Huelva, v. 19, n. 38, p. 10-12, mar. 2012. Disponible en <http://www. revistacomunicar.com>. Acceso el: 31 ene. 2014.

HABERMAS, J. Ciencia y técnica como “ideología”. Madrid: Tecnos, 1986.

HOBSBAWM, E. Naciones y nacionalismo desde 1780. Barcelona: Crítica, 1998.

KANT, I. Crítica de la razón práctica. Buenos Aires: Losada, 2008.

KUBRICK, S. La naranja mecánica. Reino Unido: Warner Bros. Pictures, 1971. 136 minutos.

LEMARCHAND, G. (Ed.). Sistemas nacionales de ciencia, tecnología e innovación de América Latina y el Caribe. Montevideo: UNESCO, 2010.

MACKNESS, J.; MAK, S.; WILLIAMS, R. The ideals and reality of participating in a MOOC. $7^{\text {th }}$ International Conference on Networked Learning Conference, University of Lancaster, p. 266-275, may. 3 y 4 de 2010. Disponible en: <http://www.lancaster.ac.uk/fss/ organisations/netlc/past/nlc2010/abstracts/PDFs/Mackness.pdf > . Acceso el: 5 mar. 2014.

MARCUSE, H. El hombre unidimensional. Ensayo sobre la ideología de la sociedad industrial avanzada. Barcelona: Planeta-Agostini, 1993. 
MARTÍN-BARBERO, J. Cuando la tecnología deja de ser una ayuda didáctica para convertirse en mediación cultural. Teoría de la Educación: Educación y Cultura en la Sociedad de la Información, Salamanca, v. 10, n. 1, p. 19-31, 2009. Disponible en: <http:// campus.usal.es/-teoriaeducacion/rev numero $10 \quad 01 / \mathrm{n} 10 \quad 01$ martin-barbero.pdf $>$. Acceso el: 12 ene. 2014.

. La educación desde la comunicación. Bogotá: Norma, 2003.

La razón técnica desafía a la razón escolar. En: NARODOWSKI, M.; OSPINA, H.; MARTÍNEZ-BOOM, A. (Eds.). La razón técnica desafía a la razón escolar. Buenos Aires: Noveduc, 2006. p. 11-25.

MARTÍNEZ-BOOM, A. Verdades y mentiras sobre la escuela. Bogotá: IDEP, 2012.

MCCLOSKEY, E. Docentes globales: un modelo para el desarrollo de la competencia intercultural on-line. Comunicar, Huelva, v. 19, n. 38, p. 41-49, mar. 2012. Disponible en: <http://www.revistacomunicar.com>. Acceso el: 1 feb. 2014.

MOUFFE, C. En torno a lo político. Buenos Aires: Fondo de Cultura Económica, 2007.

NOGUERA, C. El gobierno pedagógico. Del arte de educar a las tradiciones pedagógicas. Bogotá: Siglo Del Hombre Editores, 2012.

ORGANIZACIÓN PARA LA COOPERACIÓN Y EL DESARROLLO ECONÓMICOS (OCDE). Manual de Frascati. Medición de las actividades científicas y tecnológicas. Propuesta de norma práctica para encuestas de investigación y desarrollo experimental. Fundación Española Ciencia y Tecnología, 2002.

ORGANISATION FOR ECONOMIC CO-OPERATION AND DEVELOPMENT (OECD). Better skills, better jobs, better lives. A strategic approach to skills policies. OECD Publishing, 2012. Disponible en: <https://skills.oecd.org/documents/ OECDSkillsStrategyFINALENG.pdf $>$. Acceso el: 31 mar. 2014.

OILO, D. De lo tradicional a lo virtual: las nuevas tecnologías de la información. Conferencia Mundial sobre la Educación Superior. La Educación Superior en el Siglo XXI. UNESCO, París, 5-9 oct. 1998. Disponible en: <http://www.unesco.org/education/ educprog/wche/principal/nit-s.html>. Acceso el: 9 feb. 2014.

PLATÓN. Diálogos $I V$. República. Madrid: Gredos, 1988.

POHLENZ, A. Conflictos por acceso biotecnológico de los recursos genéticos y conocimientos tradicionales en la región andino-amazónica: los casos de patentes de la maca, el yacón y la sangre de drago. Maestría en Estudios Socioambientales. Quito: FLACSO, 2009. Disponible en: <http://repositorio.flacsoandes.edu.ec/bitstream/10469/809/3/TFLACSO-2009APT. pdf>. Acceso el: 4 mar. 2014.

PRIETO, M.F.S. La aplicación de las nuevas tecnologías en la educación. Didáctica universitaria, p. 139-148, 2001. Disponible en: <http://www.tendenciaspedagogicas.com/ Articulos/2001 06 06.pdf $>$. Acceso el: 07 febr. 2014.

REICH, S. What is globalization. Four Possible Answers. Notre Dame: Kellogg, 1998.

RUEDA, R.; QUINTANA, A. Ellos vienen con el chip incorporado. Aproximación a la cultura informática escolar. Bogotá: IDEP, 2013. 
SALGANIK, L.; RYCHEN, D.; MOSER, U.; KONSTANT, J. Definición y selección de competencias. Proyectos sobre Competencias en el Contexto de la OCDE Análisis de base teórica y conceptual. Neuchatel: OCDE, 1999.

SCHALK, A. El impacto de las TIC en la educación. Relatoría de la Conferencia Internacional de Brasilia, 26-29 abr. 2010. Disponible en: <http://unesdoc.unesco.org/ images/0019/001905/190555s.pdf $>$. Acceso el: 3 abr. 2014.

SENNETT, R. El artesano. Barcelona: Anagrama, 2009.

SHAPIN, S. A social history of truth: civility and science in seventeenth-century England. Chicago: University of Chicago Press, 1994.

SKINNER, B. Tecnología de la enseñanza. Barcelona: Labor, 1973. The technology of teaching. New York: Appleton-Century-Crofts, 1968.

TARABINI, A.Y.; BONAL, X. Globalización y política educativa: los mecanismos como método de estudio. Revista de Educación, Madrid, n. 355. p. 235-255, 2011, Disponible en: <http://dialnet.unirioja.es/servlet/articulo?codigo=3638937>. Acceso el: 20 abr. 2014.

UNESCO. Conferencia Mundial sobre la Educación Superior. La educación superior en el siglo XXI. Visión y acción. Documento de trabajo. París, 5-9 oct. 1998. Disponible en: <http://unesdoc.unesco.org/images/0011/001136/113602So.pdf>. Acceso el: 3 ago. 2014.

. La educación encierra un tesoro. Informe a la UNESCO de la Comisión Internacional sobre la Educación para el Siglo XXI. Santillana: UNESCO, 1996.

. UNESCO ICT competency framework for teachers. 2011. Disponible en: < http:// unesdoc.unesco.org/images/0021/002134/213475e.pdf>. Acceso el: 5 may. 2014.

WEINDLING, P. The uses and abuses of biological technologies: Zyklon B and gas infestation between the first world war and the holocaust. History and Technology: An International Journal, v. 11, n. 2, p. 291-298, 1994. Disponible en: <http://www. tandfonline.com/doi/abs/10.1080/07341519408581867? journalCode $=$ ghat20\#. VRrUefyUdRk>. Acceso el: 12 ene. 2014.

ZAPATA-ROS, M. Enseñanza Universitaria en linea, MOOC y aprendizaje divergente. 2013. Disponible en <http://cit.uao.edu.co/docente/sites/default/files/repositorio/ Ense\%C3\%B1anza\%20universitaria\%20en\%201\%C3\%ADnea\%20-\%20Mooc\%20 y\%20aprendizaje\%20divergente.pdf> Acceso el: 2 mar. 2014.

Recibido en 8 de Abril de 2015.

Aprobado en 11 de Enero de 2016. 